\title{
Hydrogeologische Notizen
}

Online publiziert: 4. November 2020

(c) Springer-Verlag GmbH Deutschland, ein Teil von Springer Nature 2020

\section{Grundwasser in eigener Sache}

Die Redaktion behält sich vor, eingereichte Beiträge zu kürzen oder redaktionell zu verändern.

\section{Redaktionsschluss für Beiträge zu den Hydrogeologischen Notizen}

Liebe Kollegen und Kolleginnen, in den hydrogeologischen Notizen bieten wir aktuelle und für einen großen Leserkreis interessante Mitteilungen. Damit das auch weiterhin so bleibt, freuen wir uns auch auf Ihre Beiträge. Sie können zu allen Rubriken unseres Nachrichtenteils Beiträge einreichen.

Damit wir Ihre Beiträge auch zeitnah drucken können, beachten Sie bitte unsere Termine zum Redaktionsschluss:

- Heft 1/2021: 18.12.2020

- Heft 2/2021: 10.03.2021

Ihre Beiträge senden Sie bitte an:

Patricia Schüll,

E-Mail: p.schuell@gmx.de

Vielen Dank für Ihre Unterstützung!

Die Redaktion



\section{Fachsektion Hydrogeologie (FH-DGGV)}

\section{Aufruf: Grundwasseralter gesucht!}

In einem BGR-Forschungsprojekt werden derzeit alle Altersdatierungen des Grundwassers auf dem Gebiet der Bundesrepublik Deutschland gesammelt und in einer später öffentlich zugänglichen Datenbank archiviert.

Die Daten helfen zunächst bei der Suche nach einem sicheren Standort für ein Endlager für hochradioaktive Abfälle. Gemäß Standortauswahlgesetz (StandAG) ist das Antreffen von Grundwasser mit einem Alter von bis maximal 30.000 Jahren im einschlusswirksamen Gebirgsbereich oder Einlagerungsbereich als Ausschlusskriterium definiert. Der Erfassung der räumlichen Verteilung des ,jungen“ Grundwassers in Deutschland kommt daher eine wichtige Rolle zu.

Daneben sollen die Daten für weitere wissenschaftliche Fragestellungen herangezogen werden, z.B. zur Untersuchung des Einflusses verschiedener Parameter auf die Bestimmung des Grundwasseralters und der Interpretation der Tiefenlage der jungen Grundwässer in den verschiedenen hydrogeologischen Großräumen. Auch für spätere Untersuchungen zur Ausbreitung von
Schadstoffen wie Nitrat und Pestiziden sowie für die Kalibrierung von regionalen Grundwassermodellen können die Daten in Zukunft von Nutzen sein.

Um das alles möglichst flächendeckend realisieren zu können, bittet die BGR um Unterstützung und würde sich freuen, wenn vorhandene Altersdatierungen von Grundwasser und Quellen, auch historische, an uns übermittelt werden könnten. Dies können neben Tritium, Tritium-Helium, Kohlenstoff (14C), SF6 und FCKW auch weitere Isotopendaten sein. Die Datenquelle wird natürlich immer mit genannt. Neben den Altersdaten wären die Begleitdaten wichtig, z. B. Koordinaten, Entnahmetiefe, Art der Messstelle und Jahr der Beprobung. Ein fachlicher Austausch zu dem Thema ist ebenfalls immer willkommen.

Weitere Informationen werden gerne durch Frau Annika Desens und Herrn Dr. Georg Houben gegeben:

Annika Desens

annika.desens@bgr.de

Telefon: +49 511 643-2314

Georg Houben

georg.houben@bgr.de

Telefon: +49 511 643-2373

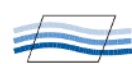




\section{Ausschreibung FH-DGGV- Geschäftsstelle}

Die Fachsektion Hydrogeologie in der DGGV e.V. ist ein Fachverband zur Förderung des wissenschaftlichen Austausches und versteht sich als Vertretung der Hydrogeologinnen und Hydrogeologen Deutschlands. Die derzeit rund 1300 Mitglieder sind in Ingenieurbüros, Industriebetrieben, Ämtern und Behörden sowie in der Wissenschaft tätig. Die Fachsektion Hydrogeologie ist Herausgeber der Zeitschrift Grundwasser, unterhält eigene Arbeitskreise, führt eine Vielzahl an Fortbildungsveranstaltungen durch und ist Veranstalter der alle 2 Jahre stattfindenden Fachtagung.

Die langjährige Leiterin der Geschäftsstelle, Frau Dr. KaufmannKnoke, wird im Laufe des Jahres 2022 in Ruhestand gehen. Wir suchen daher zum 1. Mai 2021 eine neue Geschäftsstellenleitung.

\section{Ihre Aufgaben sind:}

- Verwaltung der Mitgliederdaten: Pflege der Datenbank, Mitgliederkorrespondenz, Datenaufbereitung, Jahresrechnungen, Zahlungskontrolle

- Editorial Office der Zeitschrift Grundwasser: Kontaktstelle Editoren - Autoren - Verlag, Organisation und Kontrolle aller Abläufe für die fristgemäße Herausgabe der Fachzeitschrift

- Organisation von Fortbildungsveranstaltungen: Akquise, Kalkulation der Teilnahmegebühren, Layout der Flyer, Buchung von Hotels und $\mathrm{Ca}$ tering, Rechnungstellung

- Organisation von Tagungen: Programmplanung in Kooperation mit dem Tagungsausrichter, Buchung der Räume und des Caterings, Layout und Bestellung aller Druckerzeugnisse, Kalkulation der Teilnahmegebühren, Rechnungstellung, Betreuung der Tagungshomepage, Planung und Ausrichtung der Fach- ausstellung, Einnahmen-/Ausgabenkontrolle

- Vorstand: Vorbereitung der Vorstands- und Beiratssitzungen sowie der Mitgliederversammlungen

- Finanzen: Rechnungstellung, Kontoführung, Buchführung, Vorbereitung der Jahresabschlüsse für Steuerberater

- Öffentlichkeitsarbeit: Teilbetreuung der FH-DGGV-Internetseite, Betreuung des Informationsstandes auf Tagungen und Messen, Schriftenverkauf

Sie bringen mit:

- Abgeschlossener Hochschulabschluss, bevorzugt Fachrichtung Hydrogeologie oder vergleichbar, Promotion vorteilhaft

- Strukturierte und effiziente Arbeitsweise

- Kompetente und gewinnende Umgangsformen, Freude an der Kommunikation und an der Arbeit in Gruppen und Gremien

- Sehr gute Deutschkenntnisse, gute Englischkenntnisse

- Gute Kenntnisse in MS-Office Programmen und die Bereitschaft, sich in PC-Software einzuarbeiten

- Bereitschaft und Flexibilität bei der Durchführung von Aktivitäten der Fachsektion (auch an Wochenenden)

- Organisationsvermögen und Belastbarkeit

- Führerschein Klasse B

Wir bieten:

- Abwechslungsreiche und spannende Arbeiten in der Geschäftsstellenleitung mit vielen Gestaltungsmöglichkeiten

- Arbeitsumfeld mit kurzen Entscheidungswegen

- Fachlicher Austausch, vielfältige Kontakte, motivierendes Arbeitsumfeld

- Flexible Anstellungsbedingungen und eine leistungsgerechte Vergütung
- Gründliche und strukturierte Einarbeitung

Wir freuen uns auf Ihre Bewerbung. Wir bitten um Zusendung bis zum 28.02.2021 per E-Mail an die Adresse der Geschäftsstelle der Fachsektion Hydrogeologie in der Deutschen Geologische Gesellschaft - Geologische Vereinigung (DGGV) e.V.: geschaeftsstelle@fh-dggv.de

Ihr Traugott Scheytt (Vorsitzender der Fachsektion Hydrogeologie)

\section{Fortbildungsveranstaltung der FH-DGGV: Grundwasserhydraulik - Grundlagen der Strömung, analytische Lösungsverfahren, 25.-26.02.2021 in Bad Soden- Salmünster}

Die quantitative Beschreibung der Grundwasserströmung ist die Voraussetzung für die Beantwortung jeder hydrogeologischen Fragestellung. Der Kurs vermittelt hierzu die theoretischen Grundlagen und stellt einfache Lösungen zur Berechnung der Grundwasserströmung vor. Diese „Formeln“ erlauben eine schnelle und überschlägige Abschätzung, z.B. der Transmissivität eines Aquifers, des Grundwasserumsatzes, der Grundwasserabstandsgeschwindigkeit, der Brunnenabsenkung, des $\mathrm{Zu}-$ flusses zu einer Baugrube etc. Diese Überschlagsrechnungen sind auch noch heute im Zeitalter der numerischen Grundwassermodelle sinnvoll, da man damit sehr schnell und einfach Größenordnungen abschätzen kann. Anhand von Beispielen aus der Praxis wird die Anwendung dieser Methoden eigenständig geübt, und die Ergebnisse werden diskutiert.

Zielgruppe des Lehrgangs sind Vertreter von Ingenieurbüros, Behörden und Versorgern sowie Studierende, 
die mit hydrogeologischen Fragestellungen zu tun haben. Mathematische Grundkenntnisse sind erforderlich, und Kenntnisse in allgemeiner Hydrogeologie sind sinnvoll, allerdings will diese Fortbildungsveranstaltung insbesondere die Grundlagen vermitteln. Die Veranstaltung ist deshalb auch zur Auffrischung und Vertiefung vorhandener Kenntnisse geeignet.

Die Veranstaltung gliedert sich in folgende Einheiten:

- Theorie der Grundwasserströmung: Piezometerhöhe (BernoulliGleichung), Aquiferparameter $\left(\mathrm{k}_{\mathrm{f}}-\right.$ Wert, Transmissivität, spezifischer Speicherkoeffizient, Speicherkoeffizient), stationäre/instationäre Strömung für den gespannten und ungespannten Aquifer, Rand- und Anfangsbedingungen, Strömungsgleichung, einfache analytische Lösungen der Strömungsgleichung.

- Abschätzung der Aquiferparameter: Transmissivität, $\mathrm{k}_{\mathrm{f}}$-Wert, Speicherkoeffizient.

- Grundwassergleichen: Konstruktion und Interpretation, Stromlinien/ Bahnlinien, Strömungsnetze, Filterund Abstandsgeschwindigkeit.

- Brunnen: Fassungsvermögen, Grundwasserabsenkung/-aufhöhung Einzelbrunnen/Brunnenfelder, Reichweite der Grundwasserabsenkung, Superpositionsprinzip, Brunneneinzugsgebiet, n-Tage-Linie.

- Salz-/Süßwasser-Interaktion: Ghyben-Herzberg-Modell.

- Bauen im Grundwasser: Berechnung des Grundwasserandrangs in Baugruben, Zufluss zu Sickerschlitzen, Grundwasseraufstau durch unterirdische Bauwerke.

\section{Referenten}

Prof. Dr. Traugott Scheytt, TU Bergakademie Freiberg, ist Dozent an der TU Bergakademie Freiberg, Lehrstuhl für Hydrogeologie/Hydrochemie. Herr Scheytt hält Vorlesungen zur Hydraulik, zur Hydrochemie und zum Stoff- transport, betreut Masterarbeiten und Dissertationen und ist durch Publikationen und Vorträge wissenschaftlich ausgewiesen.

Prof. Dr. Randolf Rausch, Technische Universität Darmstadt, ist Dozent an der Technischen Universität Darmstadt (Institut für Angewandte Geowissenschaften), Mitarbeiter von BoSS Consult Stuttgart, sowie Mitglied des Water Quality Management Experts-Networks der bw-engineers. Randolf Rausch ist seit Jahrzehnten national und international in Forschung, Lehre und Consulting tätig. Schwerpunkte seiner Arbeit sind die Grundwassermodellierung sowie die Hydrogeologie arider Gebiete.

\section{Informationen}

Anmeldeschluss ist der 25. Januar 2021. Die Teilnehmerzahl ist auf 25 Personen begrenzt.

Die Teilnahmegebühr beträgt $580 €$ (Mitglieder der FH-DGGV $500 €$ ). Studentische Mitglieder zahlen $380 €$ (Mitglieder der FH-DGGV $320 €$ ). Diese Gebühren beinhalten die Kursgebühr, Veranstaltungsunterlagen sowie die Übernachtung in einem Tagungshotel einschließlich Vollpension in Bad Soden-Salmünster. Die Veranstaltung beginnt am 25.02.2021 um 10.00 Uhr und endet am 26.02.2021 gegen $13.00 \mathrm{Uhr}$.

Detaillierte Informationen entnehmen Sie bitte dem Flyer zur Veranstaltung bzw. der Internet-Seite der FHDGGV (www.fh-dggv.de).

\section{Kontaktadresse}

Anmeldung über die Geschäftsstelle der FH-DGGV:

Frau Dr. R. Kaufmann-Knoke

Telefon: +49 6321 484-784

Telefax: +496321 484-783

E-Mail: geschaeftsstelle@fh-dggv.de Internet: www.fh-dggv.de

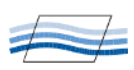

Fortbildungsveranstaltung der FH-DGGV: Hydrogeochemische Systeme quantifizieren mit PHREEQC, 08.-10.03.2021 in Hildesheim

Dieser Kurs befasst sich mit anwendungsnahen Simulationen zum Verständnis und zur Quantifizierung hydrogeochemischer Systeme. Die Tatsache, dass die Betrachtung und Bewertung der Umwelt innerhalb eines regulatorischen Rahmens durchgeführt werden, ist für das Verständnis der Rolle der geochemischen Modellierung und der Geochemie im Allgemeinen von wesentlicher Bedeutung. An dieser Stelle spielt gerade auch die Qualitätssicherung eine bedeutende Rolle. Um die beste Wissenschaft und die genausten Modelle in die Hände verantwortungsbewusster Anwender zu legen, wird die derzeitige Praxis basierend auf dem Stand der Technik der geochemischen Modellierung vermittelt. In dem Rahmen werden die Nützlichkeit und Einschränkungen der Modellkonzepte diskutiert und der ganzheitliche Blick auf geochemische Modelle geschult. Es ist das Ziel dieses Kurses, die akademische Forschung mit der Umweltpraxis $\mathrm{zu}$ verbinden und so praktizierenden Geochemikern, Hydrogeologen, Ingenieuren und Aufsichtsbehörden einen Leitfaden zur geochemischen Modellierung zu bieten.

Die Teilnehmer des Kurses erlernen die Nutzung des anwenderfreundlichen Computerprogramms PHREEQC. Dabei liegt der Fokus zunächst auf dem geochemischen Hintergrundwissen und der Rolle geochemischer Modellierung in der Praxis. Bei fast allen feldbezogenen Umweltanalysen besteht der Bedarf an Kenntnissen und/oder Vorhersagen der Konzentrationen gelöster Stoffe in Raum und Zeit mit Bezug zur Ressource Grundwasser oder in diesem Zusammenhang auftretenden Kontaminationsproblemen. Neben den Modellierungskonzepten, den thermodynamischen und 
kinetischen Grundlagen, werden Speziations- und Reaktionspfad und auch gekoppelte reaktive Transportmodelle im Kurs behandelt.

\section{Referent}

Michael Kühn hat Chemie und Geologie studiert. Er wurde 1997 an der Universität Bremen in Geochemie promoviert und hat sich 2003 an der TU Hamburg-Harburg für das Fach Hydrologie habilitiert. Michael Kühn ist Direktor des Departments Geochemie am Deutschen GeoForschungsZentrum GFZ in Potsdam und leitet dort die Abteilung Fluidsystemmodellierung. Gleichzeitig ist er Professor für Hydrogeologie an der Universität Potsdam. Sein Forschungsschwerpunkt liegt auf der Untersuchung des Verhaltens von Wasser und anderen Fluiden in den Sedimenten und Gesteinen des geologischen Untergrunds. Er quantifiziert insbesondere geochemische Fluid-Gesteins-Wechselwirkungen mit Simulationen gekoppelter Prozesse. Ziel ist die quantitative Bewertung von Fluidsystemen im Rahmen der Erkundung und der Nutzung von Georessourcen im unterirdischen Raum, auch unter Berücksichtigung ökologischer und ökonomischer Aspekte. Dabei stellt die Modellierung mit der Software PHREEQC ein wesentliches Hilfsmittel dar zum Verständnis aquatischer Systeme.

\section{Informationen}

Anmeldeschluss ist der 5. Februar 2021. Die Teilnehmerzahl ist auf 20 Personen begrenzt.

Die Teilnahmegebühr beträgt $1050 €$ (Mitglieder der FH-DGGV $930 €$ ). Studentische Mitglieder zahlen $710 €$ (Mitglieder der FH-DGGV $600 €$ ). Diese Gebühren beinhalten die Kursgebühr, Veranstaltungsunterlagen sowie die Übernachtungen in einem Tagungshotel einschließlich Vollpension in Hildesheim. Die Veranstaltung beginnt am 08.03.2021 um 9.00 Uhr und endet am 10.03.2021 gegen 17.30 Uhr.
Detaillierte Informationen entnehmen Sie bitte dem Flyer zur Veranstaltung bzw. der Internet-Seite der FHDGGV (www.fh-dggv.de).

\section{Kontaktadresse}

Anmeldung über die Geschäftsstelle der FH-DGGV:

Frau Dr. R. Kaufmann-Knoke

Telefon: +49 6321 484-784

Telefax: +49 6321 484-783

E-Mail: geschaeftsstelle@fh-dggv.de Internet: www.fh-dggv.de

\section{Fortbildungsveranstaltung der FH-DGGV: Angewandte Grundwassermodellierung IV - Kalibration und Parameteroptimierung, 14.-17.04.2021, Bad Soden- Salmünster}

Die Prognosefähigkeit von Grundwassermodellen ist - neben einem repräsentativen Hydrogeologischen Modell - im Wesentlichen bestimmt durch die Güte der Kalibration. Der wichtigste Schritt bei der Kalibration besteht in der Festlegung der maßgeblichen Volumenströme, die für die Eindeutigkeit der Modellergebnisse erforderlich ist. Im zweiten Schritt, der inversen Modellierung, geht es um die Optimierung der Modellparameter durch eine Anpassung der berechneten an die gemessenen Beobachtungsgrößen. Dabei treten Probleme bzgl. der Eindeutigkeit von räumlichen Verteilungen oder Parameterkombinationen auf und ebenso bzgl. der Genauigkeit von Ergebnissen, die von der Repräsentativität der Messungen abhängt. Im dritten Schritt wird die Belastbarkeit des kalibrierten Modells mittels einer systematischen Sensitivitätsanalyse untersucht. Dies ist für eine transparente Dokumentation und nachvollziehbare Bewertung des Modells unerlässlich.
Als Fortsetzung der Kurse zur Angewandten Grundwassermodellierung I und II und als Fortbildungsmöglichkeit für Modellierer mit Modellerfahrung bietet die FH-DGGV einen viertägigen Intensivkurs zur Kalibration und Parameteroptimierung an.

Im Kurs werden anhand speziell ausgelegter Modell-Beispiele für stationäre/instationäre Strömung und advektiven Transport die Aspekte Eindeutigkeit und Genauigkeit erläutert und quantifiziert und darauf aufbauend Strategien zur Parameteroptimierung vorgestellt. Übungen am PC vertiefen das Verständnis für die Haupteinflussfaktoren auf Kalibration und Parameteroptimierung und für die entsprechenden Schlussfolgerungen zu zulässigen Modellanwendungen und zur Belastbarkeit der Modellprognosen.

Unter Anderem werden folgende Punkte angesprochen:

- Inverse Modellierung

- Interpretation von Scatterplots und Fehlerkarten

- Automatische Parameteroptimierung mit PEST

- Quantifizierung von Eindeutigkeit und Genauigkeit

- Gewichtungsstrategien für räumliche und zeitliche Messwerte

- Optimierungsstrategien und -sequenzen

- Parameter- und Messwert-Sensitivität

- Natürliche Streuung und systematische Abweichungen

Teilnahmevoraussetzung sind Kenntnisse in der Strömungs- und Transportmodellierung. Als Programmsystem wird das frei verfügbare PMWIN verwendet. Für den für advektiven/ dispersiven Transport wird die filebasierte Parameteroptimierung mit PEST eingeführt. Damit werden die Optimierungsmöglichkeiten über den von der Modellierungs-Software vorgegebenen Rahmen hinaus erweitert. Gleichzeitig werden aber auch die Grenzen einer automatisierten Optimierung aufgezeigt und diskutiert. 


\section{Referenten}

Dr. Johannes Riegger, Institut für Wasser- und Umweltmodellierung der Universität Stuttgart, Lehrstuhl „Hydrologie und Geohydrologie“, gibt Kurse in Geohydraulik, Grundwassermodellierung, hydrologischer Modellierung, Geostatistik und GIS.

Dr. Anneli Guthke, Institut für Wasser- und Umweltsystemmodellierung der Universität Stuttgart, Lehrstuhl „Stochastische Simulation und Sicherheitsforschung für Hydrosysteme“, unterrichtet in Grundwassermodellierung und Statistik.

\section{Informationen}

Anmeldeschluss ist der 15. März 2021. Die Teilnehmerzahl ist auf $\mathbf{1 4}$ Personen begrenzt.

Die Teilnahmegebühr beträgt $1420 €$ (Mitglieder der FH-DGGV $1210 €)$. Studentische Mitglieder zahlen $920 €$ (Mitglieder der FH-DGGV $780 €)$. Diese Gebühren beinhalten die Kursgebühr, Veranstaltungsunterlagen sowie die Übernachtungen in einem Tagungshotel einschließlich Vollpension in Bad Soden-Salmünster. Die Veranstaltung beginnt am 14.04.2021 um 8.30 Uhr und endet am 17.04.2021 gegen 15.00 Uhr.

Detaillierte Informationen entnehmen Sie bitte dem Flyer zur Veranstaltung bzw. der Internet-Seite der FHDGGV (www.fh-dggv.de).

\section{Kontaktadresse}

Anmeldung über die Geschäftsstelle der FH-DGGV:

Frau Dr. R. Kaufmann-Knoke

Telefon: +49 6321 484-784

Telefax: +49 6321 484-783

E-Mail: geschaeftsstelle@fh-dggv.de Internet: www.fh-dggv.de

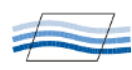

Fortbildungsveranstaltung der FH-DGGV: Durchführung und Auswertung von Pumpversuchen, 22.-24.04.2021 und 02.-04.12.2021 in Bad Soden-Salmünster

Den meisten Programmsystemen zur Pumpversuchsauswertung liegen die vereinfachenden Annahmen eines idealisierten Brunnens und eines homogenen, isotropen, unendlich ausgedehnten Grundwasserleiters zugrunde. In der Praxis enthalten die Datensätze jedoch Störeffekte des Brunnens sowie die Einflüsse überlagerter Volumenströme, natürlicher Parametervariabilität und der räumlichen Begrenzung des Grundwasserleiters.

Mit den "Diagnostischen Plots“ steht heute eine Analysemethode zur Verfügung, die aufgrund ihrer größeren Sensitivität die Identifizierung verschiedener Fließphasen und damit eine sicherere Bestimmung des passenden Aquifermodells und der zugehörigen hydraulischen Parameter ermöglicht.

Der zweitägige Kurs bietet eine intensive Einführung in die Pumpversuchsauswertung mit „Diagnostischen Plots“. Im Kurs werden hierfür einfache Excel-basierte Tabellenkalkulationen und Diagramme verwendet. Übungen am PC vertiefen das Verständnis für die Dateninterpretation insbesondere für die Ansprache von Aquifertypen, Rändern, Schichtkopplungen, Heterogenitäten und regionalen Trends.

Der technische Teil des Kurses befasst sich mit der praktischen Vorbereitung und Durchführung von Pumpversuchen mit dem Ziel einer hohen Datenqualität und eines effizienten Arbeitsablaufs.

Angesprochen und in Übungen vertieft wird die Versuchsplanung mit einer adäquaten Auslegung des Messprogramms, der technischen Komponenten und der Kostenkalkulation.
Der Kurs setzt keine Kenntnisse in der Pumpversuchsauswertung voraus, Grundkenntnisse in der Hydrogeologie und im Umgang mit Excel werden jedoch vorausgesetzt. Durch die behandelten Auswertemethoden bietet sich der Kurs auch für eine Auffrischung bzw. Vertiefung der bisherigen Erfahrung an. Die Teilnehmer erhalten ein Zertifikat.

\section{Referenten}

Prof. Dr. Uwe Hekel ist Leiter des Fachbereichs Grundwasser bei der HPC AG in Rottenburg sowie Lehrbeauftragter am Geowissenschaftlichen Institut der Universität Tübingen.

Dr. Johannes Riegger, Institut für Wasser- und Umweltmodellierung der Universität Stuttgart, Lehrstuhl „Hydrologie und Geohydrologie“, gibt Kurse in Geohydraulik, Grundwassermodellierung, hydrologischer Modellierung, Geostatistik und GIS

\section{Informationen}

Anmeldeschluss ist der 22.03.2021 (für den Termin 22.-24.04.2021) bzw. der 02.11.2021 (für den Termin 02.04.12.2021).

Die Teilnehmerzahl ist jeweils auf 16 Personen begrenzt. Die Teilnahmegebühr beträgt $970 €$ (für Mitglieder der FH-DGGV $830 €$ ). Studentische Mitglieder zahlen $630 €$ (Mitglieder der FH-DGGV $540 €$ ). Diese Gebühren beinhalten die Veranstaltungsunterlagen sowie die Übernachtung in einem Tagungshotel einschließlich Vollpension. Die Veranstaltung beginnt am 22.04.2021 (bzw. 02.12.2021) um 14.00 Uhr und endet am 24.04.2021 (bzw. 04.12.2021) gegen $15.00 \mathrm{Uhr}$.

Detaillierte Informationen entnehmen Sie bitte dem Faltblatt zur Veranstaltung bzw. der Internet-Seite der FH-DGGV (www.fh-dggv.de). 


\section{Kontaktadresse}

Anmeldung über die Geschäftsstelle der FH-DGGV:

Frau Dr. R. Kaufmann-Knoke

Telefon: +49 6321 484-784

Telefax: +496321 484-783

E-Mail: geschaeftsstelle@fh-dggv.de Internet: www.fh-dggv.de

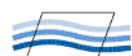

Deutsches Chapter der IAH (IAH-D)

\section{IAH-Mitgliederversammlung 2020}

Am 23.09.2020 fand die Jahresmitgliederversammlung (Annual General Meeting) der International Association of Hydrogeologists (IAH) statt. Die ursprünglich im Rahmen des 47. IAH-Kongresses in Brasilien geplante Mitgliederversammlung wurde dieses Jahr das erste Mal als Online-Treffen organisiert, da der IAH-Kongress Pandemie-bedingt auf 2021 verschoben werden musste.

Nach der Begrüßung durch den scheidenden IAH-Präsidenten Antonio Chambel und der Abnahme des Protokolls der IAH-Mitgliederversammlung von Malaga, Spanien 2019, folgten die Berichte des Vorstands für das Kalenderjahr 2019. Die IAH hatte Ende 2019 weltweit 4458 Mitglieder, einschl. 534 Studierende (davon ca. 135 Mitglieder in Deutschland) und verzeichnete damit insbesondere aufgrund des sehr erfolgreichen Kongresses in Malaga einen großen Mitgliederzuwachs. Finanziell schloss die IAH das Jahr 2019 mit einem Plus von ca. $11.000 € \mathrm{ab}$.

Auf fachlicher Ebene wurde besonders die hervorragende Qualität des Hydrogeology Journals unterstrichen. Die Zeitschrift hat sich in den letzten Jahren unter Executive Editor Cliff Voss in den wissenschaftlichen Indices sowie bei der Zahl der Beiträge weiter verbessert.

Inhaltlich zeichnet sich die IAH international zudem durch eine Vielzahl von 15 aktiven Fachkommissionen und thematischen Netzwerken aus. Hervorgehoben wurde besonders das starke Engagement der jüngeren Generation, die mit dem Early Carrier Hydrogeologists' Network (ECHN) immer wieder neue Ideen einbringt. Im letzten Jahr sind zwei weitere Kommissionen dazugekommen: die Commission on Groundwater Quality und das Socio-Hydrogeology Network.

Bei den aktuellen Entwicklungen der Mitgliederzahlen seit Beginn 2020 musste allerdings eingeräumt werden, dass der positive Trend der letzten Jahre - vermutlich auch Pandemie-bedingt - abrupt zu einem Ende kommt und aktuell ein Rückgang der Mitgliederzahlen von ca. $10 \%$ zu verzeichnen ist. Die finanzielle Situation wurde grundsätzlich als gut bewertet; die zukünftige Entwicklung der IAH als in Großbritannien registriertem Verband sei aber mit dem Austritt Großbritanniens aus der EU nicht risikofrei. Für das nächste Jahr wurde daher beschlossen, die aktuellen Mitgliedsbeiträge unverändert zu lassen und nicht zu erhöhen.

Die diesjährigen Auszeichnungen der IAH gingen an

- Presidents' Award: Bruce Misstear (IRL)

- Honorary Membership Award: Ken Howard (CAN), Cliff Voss (USA)

- Applied Hydrogeology Award: David Ball (IRL), Abdel Gadelmulal (SDN)

Der Distinguished Hydrogeology Award soll im Rahmen des 47. IAH Kongresses 2021 in Brasilien vergeben werden.

Zwischen Juli und September 2020 fanden mit einer Wahlbeteiligung von ca. $25 \%$ der Mitglieder die online abgehaltenen Wahlen zum neuen Vorstand der IAH (Council Election) für 2020-2024 statt. Dabei wurde Dave Kreamer (USA) als neuer IAH-Prä- sident und Nachfolger von Antonio Chambel (POR) gewählt, Jane Dottridge (GBR) zur Generalsekretärin, Ralf Klingbeil (DEU) zum Vice President, Programme and Science Coordination sowie Teodóra Szöcs (HUN) als Vice President, Finance and Membership bestätigt. Außerdem wurden acht weitere regionale Vice Presidents gewählt.

Zum Abschluss wurde noch der aktuelle Stand der Entwicklungen zum auf 2021 verschobenen 47. IAH-Kongress in Sao Paulo, Brasilien (22.27.08.2021) und zum kurz darauffolgenden 48. IAH Kongress in Brüssel, Belgien (06.-10.09.2021) vorgestellt. Weitere Informationen stehen auf der Webseite der IAH, www.iah.org zur Verfügung.

R. Klingbeil, Hannover iah.klingbeil@gmx.de

\section{Deutsche Vereinigung für Wasserwirtschaft, Abwasser und Abfall e.V. (DWA)}

\section{Stellungnahme zur Ackerbaustrategie 2035}

Das Bundesministerium für Ernährung und Landwirtschaft (BMEL) hat den Entwurf einer ,Ackerbaustrategie 2035“ öffentlich zur Diskussion gestellt. Damit sollen mittel- bis langfristig für die Grundlagen der Ernährung in Deutschland Perspektiven für einen produktiven und vielfältigen Pflanzenbau aufgezeigt werden.

Die DWA begrüßt grundsätzlich die Initiative für eine Ackerbaustrategie 2035. Viele der darin behandelten Themenfelder sind gut strukturiert und stellen transparent die aktuelle Situation, bestehende Konfliktbereiche und künftige Handlungsstrategien dar. Die gleichmäßige Abbildung der ökolo- 
gischen, ökonomischen und sozialen Belange - wie angestrebt - gelingt nur teilweise. Die Aufnahme von klaren Zielen und Zwischenzielen wäre sinnvoll.

Die gesamte Stellungnahme: https:// de.dwa.de/files/_media/content/01_ DIE\%20DWA/Politikinformationen/ Stellungnahmen/DWA_Stellungnahme\% 20 Ackerbaustrategie $\% 202035$ 20200821\%20fin.pdf

Internet: www.dwa.de

\section{Deutscher Verein des Gas- und Wasserfachs e. V. (DVGW)}

\section{SARS-CoV-2 in der Wasserversorgung}

\section{Hintergrund und Ergebnisse}

Nach dem Ausbruch der Atemwegserkrankung COVID-19 durch eine Infektion mit dem neuartigen Coronavirus (SARS-CoV-2) in China kam es zu einer weltweiten Pandemie. Eine Übertragung von SARS-CoV-2 erfolgt nach derzeitigem Wissensstand vor allem über den direkten Kontakt zwischen Personen oder über kontaminierte Flächen. Seit bekannt ist, dass ein Teil der infizierten Personen das Virus über den Verdauungstrakt ausscheidet, wird befürchtet, dass der Erreger über den Abwasserpfad in das Wasser gelangen kann, das zur Trinkwassergewinnung genutzt wird. Aus diesem Grund wurde im Rahmen der DVGW-Forschung die Literaturstudie zu SARS-CoV-2 in der Wasserversorgung beauftragt, die den derzeitigen Stand des Wissens zum Vorkommen und Verhalten des Erregers in der aquatischen Umwelt zusammenfasst. Der vorliegende Bericht bietet eine Übersicht des aktuellen wissenschaftlichen Sachstandes und bietet Unternehmen der Wasserwirtschaft eine Grundlage für die Kommunikation in diesem Bereich.

Ein Ergebnis der Auswertung von über 300 einschlägigen Fachpublika- tionen ist, dass eine Übertragung der Krankheit über das Trinkwasser nahezu ausgeschlossen werden kann. Es ist gegenwärtig kein Fall bekannt, bei dem sich Menschen über den Verzehr kontaminierter Lebensmittel - und damit auch Trinkwasser - mit dem neuen Coronavirus infiziert haben. Außerdem zeigte die Literaturrecherche, dass das in Deutschland seit Jahrzehnten eingeführte Multi-Barrieren-System, bestehend aus Ressourcenschutz, Wassergewinnung, Wasseraufbereitung und Wasserverteilung, eine sichere Grundlage zur Vermeidung wasserbedingter Epidemien ist. Das Trinkwasser ist deshalb sehr gut gegen alle Viren geschützt.

\section{Ziele und Methodik}

Ziel der Literaturstudie zu SARS-CoV-2 in der Wasserversorgung war es, eine zitierfähige Übersicht des aktuellen wissenschaftlichen Sachstandes zu schaffen, die den Wasserversorgungsunternehmen als Grundlage für die weitere kommunikative Verwertung dienen kann. Die Schlussfolgerungen und Interpretationsansätze, die aus der komplexen Fachinformationen abgeleitet werden können, sollten zudem allgemein verständlich dargestellt werden. Hierfür wurden über $300 \mathrm{Pu}$ blikationen gesichtet, ausgewertet und in dem vorliegenden Bericht zusammengefasst.

Die Studie wurde durchgeführt von: TZW: DVGW-Technologiezentrum Wasser, Karlsruhe

Projektlaufzeit: 06/2020-08/2020

Die Literaturstudie ist Bestandteil ausgewählter Abonnements des DVGWRegelwerk Online Plus.

Bei Fragen zum Forschungsprojekt wenden Sie sich bitte an:

Dr. Mathis Keller

Hauptgeschäftsstelle/Technologie und Innovationsmanagement

Telefon: +49 228 9188-727

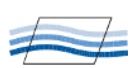

Berichte und Informationen

\section{FH-DGGV-Fortbildung in Corona-Zeiten}

Der diesjährige Fortbildungskurs der FH-DGGV „GIS-Werkzeuge für die hydrogeologische Praxis - Arbeiten mit QGIS“ stand natürlich wie vieles in dieser Zeit unter dem Einfluss von Covid19. Nicht nur die Verschiebung von Mai auf August inkl. der Absage einiger Teilnehmender aufgrund der nicht genehmigten Dienstreise, sondern auch die organisatorischen Rahmenbedingungen mussten den Auflagen angepasst werden, um die Gesundheit der Teilnehmenden und der zwei Dozenten zu schützen. Dem Landhotel Betz fiel dabei als Veranstaltungsort eine zentrale Rolle zu. Ein gut organisiertes Hygienekonzept des Landhotels Betz gab allen Teilnehmenden und Dozenten ein Gefühl größtmöglicher Sicherheit während der gesamten Kursdauer.

Die Kursinhalte entsprachen denen der letzten Jahre, wobei sich hier einmal mehr der Umstieg von streng lizenzierten auf OpenSource-Werkzeuge und kostenlose Werkzeuge bewährte. Die Pandemie-Vorschriften hatten auf den geplanten Kursverlauf nur einen sehr geringen Einfluss. Lediglich die enge Zusammenarbeit von Teilnehmenden ,an einem Tisch“ war nicht möglich. Dafür wurde trotz der geringen Teilnehmenden-Zahl ein grö-

Ein Termin, den Sie sich merken sollten:

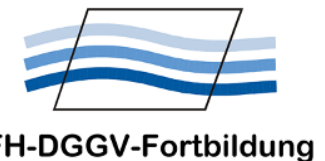

Grundwasserhydraulik

Bad Soden-Salmünster

25.02.-26.02.2021 
Berer Tagungsraum vom Haus zur Verfügung gestellt.

An zwei der drei Abende wurden kurzfristig und spontan zwei zusätzliche Themen im engeren Interessenbereich der Teilnehmenden angeboten: Anbindung geologischer Datenbanken an GIS und Zeitreihenanalyse hydrogeologischer Daten. Durch die gute Internetanbindung des Hauses konnte auch die hierfür notwendige Software schnell nachinstalliert werden. Das Angebot wurde von 4-5 Teilnehmenden gerne wahrgenommen und die Do- zenten denken darüber nach, einerseits diese Form der von Teilnehmenden gewünschten Erweiterung des Kursprogramms und die konkret in diesem Jahr gewünschten Inhalte mit in das Kursprogramm des kommenden Jahres zu übernehmen. Insgesamt also ein sehr positives Fazit des Fortbildungskurses in Covid19-Zeiten.

W. Gossel, Halle a. S.

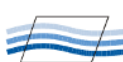

\section{Forum}

\section{Fachsektion Hydrogeologie}

\section{In eigener Sache}

Liebe Kollegen und Kolleginnen, im Forum stellen wir Stellungnahmen zu aktuellen Themen für unsere Mitglieder zur Diskussion. Gerne nehmen wir Ihre Beiträge - beispielsweise als Leserbriefe - entgegen. Wir freuen uns auf eine intensive Diskussion!

Ihre Beiträge senden Sie bitte an:

Patricia Schüll

E-Mail: p.schuell@gmx.de

Vielen Dank für Ihre Unterstützung!

Die Redaktion

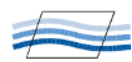

\section{Vorrang der notwendigen \\ Versorgung der Menschen mit Trinkwasser langfristig sicherstellen und Nutzungskonkurrenzen lösen}

Angesichts der letzten Hitzesommer und der resultierenden Wasserknappheit in einzelnen Kommunen hat Bundesumweltministerin Svenja Schulze eine „Nationale Wasserstrategie“ ange- kündigt, die Prioritäten und verbindliche Regelungen bei der Wassernutzung festlegen soll. Prof. Dr. Lothar Scheuer, Vorstand Aggerverband und Präsident der Allianz der öffentlichen Wasserwirtschaft e.V. (AöW) kommentiert:

„Die AöW begrüßt ausdrücklich den Vorstoß der Bundesumweltministerin für eine „Nationale Wasserstrategie“. Unbestritten ist, dass für alle Nutzergruppen in Zukunft der sorgsame Gebrauch unserer Trinkwasserressourcen noch wichtiger wird. Nutzungskonkurrenzen um Wasser müssen transparent aufgezeigt, gesamtgesellschaftlich diskutiert und letztlich politisch gelöst werden. Dabei muss die Trinkwasserversorgung der Bevölkerung als Menschenrecht an der Spitze der Prioritäten stehen und langfristig gesichert werden. Alle anderen Nutzungen müssen gegebenenfalls abgewogen werden. Damit sind bei regionaler Wasserknappheit Rasen- oder Grünflächenbewässerung ebenso auf den Prüfstand zu stellen wie eine landwirtschaftliche Bewässerung ohne eine an den Klimawandel angepasste wassersparende Bewirtschaftung. Da wir insgesamt über ausreichende Ressourcen verfügen, sind auf lokaler und regionaler Ebene Kooperationen zu prüfen und zu realisieren.

Die Wasserwirtschaft in öffentlicher Hand bietet aufgrund ihrer Ge-
Ein Termin, den Sie sich merken sollten:

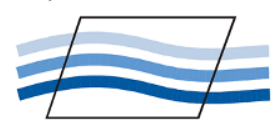

FH-DGGV-Fortbildung

Hydrogeochemische Systeme quantifizieren mit PHREEQC

Hildesheim

08.-10.03.2021

meinwohlorientierung die besten Voraussetzungen, um die Herausforderungen der notwendigen Anpassungen an den Klimawandel anzugehen. Hierfür ist es entscheidend, die öffentliche Wasserwirtschaft auch langfristig vor weiterer Liberalisierung und Privatisierung zu sichern."

Weitere Forderungen der AöW zur Anpassung an den Klimawandel finden Sie in unserem Positionspapier.

\section{Kontakt}

Presse- und Öffentlichkeitsarbeit

E-Mail: presse@aoew.de

www.aoew.de

Ein Termin, den Sie sich merken sollten:

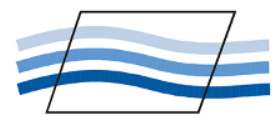

FH-DGGV-Fortbildung

GIS-Werkzeuge für die hydrogeologische Praxis

Bad Soden-Salmünster

12.-15.05.2021 


\section{Glosse}

\section{Die Selbstverleugnungs- epidemie PhD}

Mein lieber FH-Präsident, ich muss Dir heute wegen einer Auffälligkeit am Hydro-Horizont schreiben, die mir massive Sorgen bereitet. Eventuell hört es ja auch wieder auf und erreicht niemals den heiligen Hydro-Himmel. Was mich besorgt, ist wieder so ein Trend, wie ich schon viele in meiner Geokarriere erlebt, durchlebt, durchlitten, erlitten habe. Und jedes Mal kam Unsinn heraus. Vielleicht weil ein paar einzelne, Halbwissentragende-blind-und-dumm-oder-indiesem-Fall-des-Englischen-nichtmächtige Menschen voran geprescht sind? Oder waren und sind das alles eventuell narzisstisch-donald-trumpistische Karrieristen, die sich wichtigmachen wollen und irgendeiner Eitelkeit hinterherrennen, ohne sich Gedanken zu machen? Und in ein paar Jahren werden wir wieder zusammenstehen und uns kopfschüttelnd fragen: „Wie konnte das nur passieren?" So wie bei unserem guten alten, hochwertigen Diplom, das wir ohne Not durch Bätschler und Master ersetzt haben, und, wie sich nachträglich herausstellte, durch den Profilbildungszwang der einzelnen Geo-Institute, ein völliger Fehlgriff in die Toilette der Geoausbildungsgeschichte wurde. Aber ich

Ein Termin, den Sie sich merken sollten:

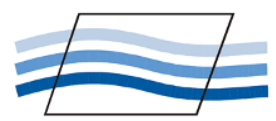

FH-DGGV-Fortbildung

Durchführung und Auswertung von Pumpversuchen

Bad Soden-Salmünster

22.-24.04.2021 schweife ab. Nun kommt immer mehr ein neuer Unsinn auf, der allerdings diesmal eventuell die JuristInnen auf den Plan ruft, weil nicht nur unsinnig, sondern bestrafungswürdig!

Ich schreibe vom $\mathrm{PhD}$ - Trend! Immer häufiger nennen sich Personen selbst Philosophical Doctor, Doctor Philosophiae, oder abgekürzt $\mathrm{PhD}$, obwohl sie nachweislich in Deutschland zum Dr. rer. nat. promoviert wurden. Oder man liest in Bewerbungen, dass jemand angeblich „... 1992 einen Master's Degree in Darmstadt an der Technical University und 1997 an der University of Kiel einen PhD ..." erhalten hätte. Oha, gab es denn diese beiden akademischen Grade zu dieser Zeit in Deutschland? Und hatten diese Unis damals schon englische Namen? Ja tatsächlich, es gab und gibt auch immer noch den Dr. Phil. (Doktor der Philosophie) in den Geowissenschaften in Deutschland! Der PhD im anglosächsischen Raum, der einem deutschen Dr. ähnlich ist, ist jedoch in der Regel an ein 4-5-jähriges, verschultes Ausbildungsprogramm gebunden (mit Vorlesungen und Prüfungen), wo zum Teil ein begabte/r BachelorIn (oder nur 1-jährige/r MasterabsolventIn) direkt in ein PhD- bzw. Doktorat-Programm gehen kann! Das stelle man sich im konservativen Deutschland vor, wo man in der Regel nur 3 Jahre lang eine $50 \%$-Stelle zur Promotion bekommt (also 50\%-Bezahlung!) und das 4. und eventuell 5. Jahr, das man regelmäßig in einer forschungsorientierten Promotion braucht, ,irgendwie“ finanziert (das ist dann immer in der Danksagung einer Dissertationsschrift nachzulesen, welche Verwandten das finanziert haben).

Der deutsche Hang zur Selbstverleugnung treibt nun immer mehr skurrile Blüten und dies obwohl ein akademischer Grad juristisch gesehen immer nur so geführt werden darf, wie dieser
Ein Termin, den Sie sich merken sollten:

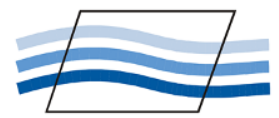

FH-DGGV-Fortbildung

Angewandte

Grundwassermodellierung IV

Bad Soden-Salmünster

14.-17.04.2021

erworben wurde. Und dieser eventuell strafrechtlich relevante Tatbestand des selbstgewandelten $\mathrm{PhD}$ (anstatt des guten Dr. Phil. oder Dr. rer. nat., den man erworben hat) wird mittlerweile sogar in englischen Ausschreibungen von deutschen Universitäten und Stipendiengebern, die sich immer mehr angloamerikanisieren wollen, völlig schmerzfrei benutzt (intern spricht man häufig von ,internationalisieren“, es bedeutet aber faktisch nur den Blick nach Trumpland)!

Ehre, wem Ehre gebührt, wer einen PhD-Grad im Ausland erworben hat, sollte (oder muss sogar) dies so anzeigen. Was aber soll diese Dr.-Selbstverleugnungepidemie, was wollen diese Personen und Institutionen damit zeigen? Ich habe keine Antwort darauf. Sicherlich aber nicht Selbstbewusstsein.

Beste Grüße Dein,

Doktor rerum naturalis, Diplom-Geologe, Master of Complete Transcendal Stupidification (MSc CTS)

C. Orinthen-Kacker, Klotzenhausen 


\title{
Literatur und Medien
}

\section{Buchbesprechungen}

\section{Geothermie}

\author{
Stober, I. \& Bucher, K. (2020): \\ Geothermie. 3. Auflage, \\ 390 S., Heidelberg; https:// \\ doi.org/10.1007/978-3-662-60940-8 \\ (Springer Verlag); Softcover $(34,99 €)$ \\ $+\operatorname{eBook}(26,99 €)$
}

Nach einer Einführung in die Grundlagen der Geothermie werden die wichtigsten thermischen und hydraulischen Parameter in ihrer Bedeutung behandelt. Das nunmehr bereits in der 3., überarbeiteten und aktualisierten Auflage vorliegende Buch gibt einen Überblick über alle derzeit verfügbaren Nutzungssysteme der oberflächennahen und tiefen Geothermie und betrachtet diese bezüglich ihrer Effizienz und potentieller Umweltauswirkungen. Neu aufgenommen in die 3. Auflage wurden geothermische Nutzungen in Hochenthalpie-Gebieten mit Beispielen aus Island, USA und Neuseeland. Im Detail besprochen werden Planung, Bau und Betrieb von Erdwärmesonden, von hydrothermalen Anlagen, von EGS-Systemen und von Hochenthalpie-Nutzungen. Spezielle

\section{Veranstaltungskalender}

\section{Tagungen und Kongresse}

\author{
Mexiko-Stadt, Mexiko \\ 11.-15.01.2021 \\ 14th International Conference on $\mathrm{Hy}$ - \\ droinformatics \\ E-Mail: contact@hic2020.org \\ Internet: hic2020.org
}

Kapitel sind der Hydraulik, d.h. der Durchführung und Auswertung hydraulischer Tests, der Hydrochemie, d.h. dem Themenbereich Lösung und Fällung, den geophysikalischen Untersuchungsmethoden an der Erdoberfläche und im Bohrloch sowie dem Abteufen und Ausbau von Tiefbohrungen gewidmet. Mit diesem Buch wird erstmalig die Geothermie in ihrer vollen Breite abgedeckt.

Die Redaktion

\section{Die Keilhacks}

Kästner, Helga \& Günter (2017): Unvergessene Belziger - Die Keilhacks. - 144 Seiten, 90 Abbildungen; ISBN 978-3-941175-754 (Treibgut Verlag Berlin); $16 €$

Der Treibgut Verlag in Berlin zeichnet sich durch eine Reihe von regional interessanten Veröffentlichungen aus. Eine davon ist das Buch „Die Keilhacks“ in der Reihe ,Unvergessene Belziger". Die Autoren Helga und Günter Kästner haben sich der Aufga- be gewidmet, die Historie dieser Familie und der Person Konrad Keilhacks aus verschiedenen Perspektiven darzustellen. Im ersten Teil wird die Familiengeschichte und der Werdegang von Konrad Keilhack anhand von Dokumenten und Bildern abgehandelt. Im zweiten Teil schreibt Hans Quitzow anhand von zahlreichen Schriftstücken, Gutachten und Veröffentlichungen über das Leben seines berühmten Großvaters. Dieses Leben ist geprägt von herausragenden beruflichen Erfolgen, aber auch von persönlichen Tragödien, wie dem kriegsbedingten Tod naher Angehöriger und dem tragischen Ableben Konrad Keilhacks in einem Bombenhagel in Berlin. Im dritten Teil berichtet Diakon Albert Baur - Vater der ersten Ehefrau von Konrad Keilhack - liebevoll über die Jugend seiner Tochter Clara.

Alles in allem handelt es sich hierbei um ein Dokument der Geschichte eines verdienten Hydrogeologen und damit auch der Hydrogeologie.

W. G. Coldewey, Münster

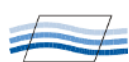

\section{Czestochowa, Polen}

25.-26.03.2021

XXIII Scientific and technical Conference - Exploitation and Risks to Water Intakes

E-Mail: kntpwik@gmail.com Internet: www.knt.czest.pl

\section{Maastricht, Niederlande 17.-20.05.2021}

LuWQ2021 - 5th International Conference on LAND USE and WATER QUALITY: Agriculture and the Environment

E-Mail:karel.kovar@rivm.nl; dico.fraters@rivm.nl Internet: luwq2021.nl 


\section{Paris, Frankreich}

26.-28.05.2021

IAH: Groundwater, Key to the Sustainable Development Goals

Internet: www.cfh-aih.fr/colloques-etvisites

\section{Dresden}

\section{Im Juni 2021}

Dresdener Grundwassertage 2021

Internet: www.dgfz.de

\section{Budapest, Ungarn}

07.-09.07.2021

IAH: International Symposium on

Geofluids

Internet: geofluids2020.hu

\section{Kiel}

14.-16.07.2021

30. Doktorandentreffen

E-Mail: adrian.metzgen@ifg.unikiel.de

\section{Sao Paulo, Brasilien}

22.-27.08.2021

47th IAH Congress

Internet: iah2021brazil.org/en/

\section{Brüssel, Belgien}

06.-10.09.2021

48. IAH Kongress

Internet: iah2021belgium.org

\section{Karlsruhe}

19.-23.09.2021

DGGV, KIT: GEOKARLSRUHE 2021: Sustainable Earth - from processes to resources

Internet: www.geokarlsruhe2021.de

\section{Fortbildungslehrgänge}

\author{
FH-DGGV \\ Telefon: +49 6321 484-784 \\ Telefax: +496321 484-783 \\ E-Mail: geschaeftstelle@fh- \\ dggv.de \\ Internet: www.fh-dggv.de
}

\subsection{2.-26.02.2021}

Grundwasserhydraulik - Grundlagen der Strömung, Analytische Lösungsverfahren in Bad Soden-Salmünster

\section{8.-10.03.2021}

Hydrogeochemische Systeme quantifizieren mit PHREEQC in Hildesheim

\section{4.-17.04.2021}

Angewandte Grundwassermodellierung IV - Kalibration und Parameteroptimierung in Bad Soden-Salmünster

\section{2.-24.04.2021}

Durchführung und Auswertung von Pumpversuchen in Bad Soden-Salmünster

\section{2.-15.05.2021}

GIS-Werkzeuge für die hydrogeologische Praxis - Arbeiten mit QGIS in Bad Soden-Salmünster

\section{7.-18.09.2021}

Immissionspumpversuche als Werkzeug zur Ermittlung von Schadstofffahnen und -frachten im Grundwasser in Bad Soden-Salmünster

\section{3.-24.09.2021}

Grundlagen und Konzepte in der organischen Hydrochemie in Fulda
10.-13.11.2021

Angewandte Grundwassermodellierung I - Einführung in die Strömungs- und Transportmodellierung in Bad Soden-Salmünster

\section{2.-04.12.2021}

Durchführung und Auswertung von Pumpversuchen in Bad Soden-Salmünster

\section{DGFZ e.V.}

Telefon: +49 351 4050-676

Telefax: +49 351 4050-679

E-Mail: weiterbildung@dgfz.de Internet: www.dgfz.de

02.02.2021

Probennahme von Abfällen nach LAGA PN98

\section{9.-10.03.2021}

Grundlagen der Hydrogeologie für Ingenieure

\subsubsection{1}

Ausbau und Benutzung von Gewässern

Hinweis des Verlags Der Verlag bleibt in Hinblick auf geografische Zuordnungen und Gebietsbezeichnungen in veröffentlichten Karten und Institutsadressen neutral. 


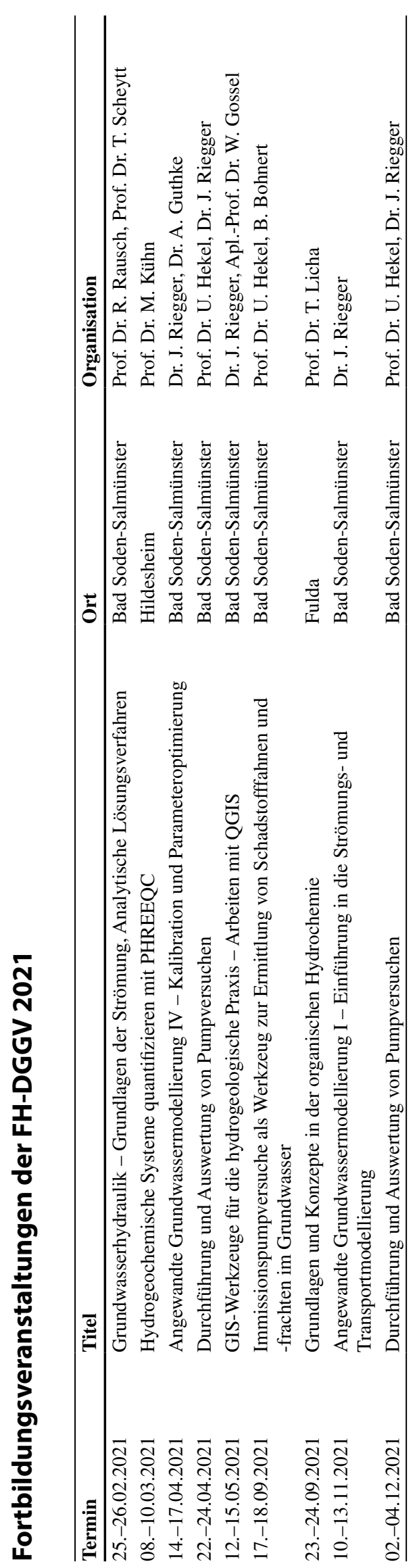

